\title{
Microscopic origin of light-induced ESR centers in undoped hydrogenated amorphous silicon
}

\author{
Takahide Umeda ${ }^{1,2, *}$ Satoshi Yamasaki, ${ }^{1}$ Junichi Isoya, ${ }^{1,3}$ and Kazunobu Tanaka ${ }^{1,2}$ \\ ${ }^{1}$ Joint Research Center for Atom Technology (JRCAT)-National Institute for Advanced Interdisciplinary Research (NAIR), \\ Tsukuba,305-8562, Japan \\ ${ }^{2}$ Institute of Materials Science, University of Tsukuba, Tsukuba, 305-0006, Japan \\ ${ }^{3}$ University of Library and Information Science, Tsukuba, 305-0821, Japan \\ (Received 12 March 1999; revised manuscript received 2 May 2000)
}

\begin{abstract}
${ }^{29} \mathrm{Si}$ hyperfine (hf) structures of light-induced electron-spin-resonance (LESR) centers of $g=2.004$ and 2.01 have been investigated in undoped hydrogenated amorphous silicon $(a-\mathrm{Si}: \mathrm{H})$ with different ${ }^{29} \mathrm{Si}$ content $(1.6$, 4.7,9.1 at. \%) by means of pulsed and multifrequency $(3,11,34 \mathrm{GHz})$ ESR techniques. We have experimentally deconvoluted overlapping LESR signals using the difference in the spin-lattice relaxation time between the two signals. The deconvoluted ${ }^{29} \mathrm{Si}$ hf structure of $g=2.004$ indicates that the wave function of the $g=2.004$ center spreads mainly over two Si atoms. Accordingly, we propose that the origin of $g=2.004$ is electrons trapped in antibonding states of weak $\mathrm{Si}-\mathrm{Si}$ bonds rather than those trapped at positively charged dangling bonds. The isotropic hf splittings were estimated to be around $7 \mathrm{mT}$ for $g=2.004$ and below $3 \mathrm{mT}$ for $g=2.01$, which are in good agreement with characteristics of the antibonding and bonding states of the weak Si-Si bond. We suggest, from our ${ }^{29} \mathrm{Si}$ hf data and other experimental findings, that the $g=2.004$ center is localized spatially more than conduction-band-tail electrons detected by photoluminescence.
\end{abstract}

\section{INTRODUCTION}

In amorphous semiconductors, there exist band-tail states in the gap both just below the conduction band and above the valence band, which have been evidenced by the optical absorption spectrum, ${ }^{1}$ photoemission spectra, ${ }^{2}$ drift mobilitymultiple trapping, ${ }^{3}$ and modulated photocurrent spectroscopy. ${ }^{4}$ The band-tail states are characterized as localized states and play an important role in transport and optical properties. It is widely accepted that those localized states originate from potential fluctuations due to variation in bond lengths, bond angles, and other topological parameters. However, there has been no experimental information available on the microscopic structure of the potential fluctuation.

In undoped $a-\mathrm{Si}: \mathrm{H}$, two light-induced electron-spinresonance (LESR) signals of $g=2.004$ and 2.01 have been detected at low temperatures. ${ }^{5}$ Doped $a$-Si:H, e.g., phosphorus- and boron-doped $a$-Si:H, also shows $g=2.004$ and 2.01 signals in the dark at low temperatures. In these samples the Fermi level shifts up to the conduction- and down to the valence-band tail in thermal equilibrium. ${ }^{6-8}$ On the basis of doping, photoluminescence, and photoconductivity experiments, Street, Biegelsen, and Wiesfield concluded that the origins of those signals should be ascribed to conduction-band-tail electrons $(g=2.004)$ and valenceband-tail holes $(g=2.01) .^{7}$ Thus, the microscopic origins of these ESR centers are directly connected to microscopic information about the potential fluctuation of the band-tail states.

The microscopic origin of the LESR centers is also important to another unresolved issue: whether undoped $a-\mathrm{Si}: \mathrm{H}$ films include a large number of charged dangling bonds (DB's) or not at thermal equilibrium. For example, chalcogenide glasses also show LESR signals at low temperatures, but almost no ESR signals are detected in the dark. ${ }^{9}$ This has been interpreted in terms of negative effective correlation energy $(U)$ for DB's, that is all DB's are considered to form pairs of positively and negatively charged DB's in thermal equilibrium. $^{9-11}$ In this model, naturally, the origins of the LESR signals are photoexcited electrons and holes trapped at the charged DB's. ${ }^{9}$ In contrast, $a-\mathrm{Si}: \mathrm{H}$ shows a pronounced dark ESR signal of $g=2.0055$ that has been generally identified as a neutral DB signal. ${ }^{12-14}$ It is therefore believed that $a-\mathrm{Si}: \mathrm{H}$ is a positive- $U$ material. ${ }^{15}$ Further, this assignment is consistent with the sensitivity of the Fermi level to doping in $a$-Si:H. ${ }^{8,16}$ However, several papers have argued the existence of a large number of charged DB's in thermal equilibrium, which is associated with the coexistence of negative and positive $U \cdot{ }^{17-20}$ In those papers, a carrier-trapped negative- $U \mathrm{DB}$ is one of the candidates for the origin of the LESR centers and possibly for the precursor of photocreated neutral DB's in the Staebler-Wronski effect. ${ }^{17-20}$ Thus the LESR centers are related to a major unresolved problem in $a-\mathrm{Si}: \mathrm{H}$.

The microscopic origins of the 2.004 and 2.01 centers are expected to be revealed by detailed analyses of hyperfine (hf) structures of ${ }^{29} \mathrm{Si}$ (nuclear spin of $I=\frac{1}{2}$, natural abundance of 4.7 at. \%) in the ESR spectrum. Yamasaki et al. detected such ${ }^{29} \mathrm{Sihf}$ structures in the LESR spectrum of undoped $a$-Si:H using a pulsed ESR technique. ${ }^{19}$ However, it was difficult to experimentally deconvolute the two overlapping LESR signals, which is necessary for extracting conclusive information about the ${ }^{29} \mathrm{Si}$ hf interactions. On the other hand, no discernible ${ }^{29} \mathrm{Sihf}$ structure has been found in doped $a$-Si:H owing to the interference with hf structures of the dopant atoms. ${ }^{11,21}$

Therefore, we directed our attention to the experimental deconvolution of ${ }^{29} \mathrm{Si}$ hf structures of LESR spectra in undoped $a-\mathrm{Si}: \mathrm{H}$. We found a difference in the spin-lattice relaxation time $\left(T_{1}\right)$ between two LESR signals, which enables us to deconvolute the LESR signals experimentally. For the purpose of precise analysis of ${ }^{29} \mathrm{Sihf}$ interactions, 
TABLE I. List of samples and their properties. Total and distant hydrogen content was determined by ${ }^{1} \mathrm{H}$ NMR measurements. The distant hydrogen was revealed by a narrow, Lorentzian component (full width at half maximum $10 \mathrm{kHz}$ ) in the ${ }^{1} \mathrm{H} N \mathrm{NM}$ spectrum. Activation energy of the dark conductivity of the B-doped sample $\left(E_{a}\right)$ was deduced from our previous data. (Ref. 23)

\begin{tabular}{lccc}
\hline \hline \multicolumn{4}{c}{ Undoped } \\
$\begin{array}{l}\left.{ }^{29} \mathrm{Si}\right] \\
(\text { at. } \%)\end{array}$ & $\begin{array}{c}\text { Total }\left[{ }^{1} \mathrm{H}\right] \\
(\text { at. \%) }\end{array}$ & $\begin{array}{c}\text { Distant }\left[{ }^{1} \mathrm{H}\right] \\
(\text { at. } \%)\end{array}$ & $\begin{array}{c}N_{\text {dark }} \\
\left(\mathrm{cm}^{-3}\right)\end{array}$ \\
\hline 1.6 & 8.9 & 2.2 & $6 \times 10^{15}$ \\
4.7 & 6.8 & 1.1 & $1 \times 10^{15}$ \\
9.1 & 10.7 & 3.3 & $4 \times 10^{15}$ \\
& & B doped & \\
{$\left[\mathrm{B}_{2} \mathrm{H}_{6}\right] /\left[\mathrm{SiH}_{4}\right]=0.89 \%$} & & $E_{a}=0.25-0.3 \mathrm{eV}$ \\
\hline \hline
\end{tabular}

undoped samples with different amounts of the ${ }^{29} \mathrm{Si}$ isotope $(9.1,4.7,1.6$ at. \%) were prepared and subjected to pulsed ESR measurements. Prior to this paper, we published a brief report of our work. ${ }^{22}$ This paper presents more detailed data and complete descriptions of the experiments and discussion. After the detailed description of experimental conditions (Sec. II), the spin-lattice relaxation of the LESR centers is elucidated by time-domain measurements of pulsed ESR (Sec. III A). Then, taking advantage of the difference in $T_{1}$, experimentally deconvoluted spectra of $g=2.004$ and 2.01 are presented (Sec. III B). From ${ }^{29} \mathrm{Si}$ hf structures of the deconvoluted 2.004 and 2.01 spectra, detailed information about the electronic structures of the LESR centers is obtained (Sec. III C). Finally, we propose a weak Si-Si bond model to account for the origin of the LESR centers (Sec. IV A), and discuss this model in comparison with previous photoluminescence data (Sec. IV B).

\section{EXPERIMENTAL DETAILS}

Undoped $a-\mathrm{Si}: \mathrm{H}$ samples were deposited by the conventional rf glow-discharge technique on aluminum foil at $250{ }^{\circ} \mathrm{C}$. The ratio of ${ }^{29} \mathrm{Si}$ atoms to total $\mathrm{Si}$ atoms, $p$, was varied from 1.6 to 4.7 to 9.1 at. \% for our samples. Flaked samples ( 1 to $2 \mu \mathrm{m}$ in thickness, around $30 \mathrm{mg}$ ) were collected in a high-purity vitreous-silica tube $(2.5 \mathrm{~mm}$ in inner diameter) for ESR measurements. We also prepared a borondoped $a$-Si:H sample from a mixture of $\mathrm{SiH}_{4}$ and $\mathrm{B}_{2} \mathrm{H}_{6}$ with $\left[\mathrm{B}_{2} \mathrm{H}_{6}\right] /\left[\mathrm{SiH}_{4}\right]=0.89 \%$ at the deposition temperature of $250{ }^{\circ} \mathrm{C}$. Dark spin densities $\left(N_{\text {dark }}\right)$ and hydrogen contents of our samples are listed in Table I.

ESR spectra were measured by the echo-detected ESR technique of pulsed ESR to perform a variety of deconvolution procedures and to obtain wide-dynamic-range spectra. In the standard technique of echo-detected ESR, the spectrum is obtained by recording amplitudes of the two-pulse Hahn spin echo $\left(90^{\circ}\right.$ pulse $-\tau-180^{\circ}$ pulse- $\tau-$ echo, $\tau$ $=240 \mathrm{nsec}$ ) as a function of magnetic-field strength. ${ }^{19}$ At each magnetic field, a pulse sequence was repeated 1000 to 4000 times with an interval of RT (repetition time) which is generally set to be much longer than $T_{1}$. The $T_{1}$ value represents a characteristic time for the spin system to recover its equilibrium state completely. The magnetic-field increments were $0.04 \mathrm{mT}$ for the center part of the spectrum with a $5 \mathrm{mT}$ width and $0.04-0.15 \mathrm{mT}$ for the remaining part. The echodetected ESR spectrum is equivalent to the absorption spectrum of conventional continuous-wave (cw) ESR, although the baselines of echo-detected ESR spectra are much flatter than those of cw ESR spectra. ${ }^{19,24}$ This enables us to observe clear ${ }^{29} \mathrm{Sihf}$ structures of the LESR spectrum. All echodetected ESR spectra were measured with a home-built pulsed ESR spectrometer with a microwave frequency of 11 GHz. This frequency was more useful for the deconvolution than the usual $9.2 \mathrm{GHz}$ ( $X$ band), because overlapping of the two signals of $g=2.004$ and 2.01 decreases as the resonant microwave frequency increases. ${ }^{6,22}$ The details of our pulsed ESR spectrometer were described in previous papers. ${ }^{24,25}$ The spin echo was generated by microwave pulses 17 and 34 nsec wide for $90^{\circ}$ and $180^{\circ}$ pulses, respectively, whose magnetic-field strength was estimated to be about $0.52 \mathrm{mT}$. The echo intensity was accumulated with a 50-nsec-wide boxcar gate and was then digitized with a 12-bit analog-todigital converter.

Sample temperatures were controlled from 4.5 to $300 \mathrm{~K}$ using an Oxford ESR-900 system, and were measured by a thermocouple in the sample tube. The LESR intensity increases monotonically with decreasing temperature in addition to the contribution of the Boltzmann factor. ${ }^{19}$ On the other hand, the number of echo signals accumulated was limited by the long repetition time due to a rapid increase in $T_{1}$. The best temperature for getting a high signal-to-noise ratio was found to be around $30 \mathrm{~K}$.

LESR measurements were carried out using a Ti-sapphire laser $(h \nu=1.7 \mathrm{eV})$. The laser light was radiated into a microwave cavity through a glass fiber. The light intensity on the sample was adjusted to be $30 \mathrm{~mW} / \mathrm{cm}^{2}$, unless noted otherwise. We checked the time evolution of the LESR spectra and confirmed that there was no appreciable influence of photodegradation on any LESR spectrum.

Some of the ESR spectra were measured using a cw ESR spectrometer (Bruker ESP300E) with microwave frequencies of 3 and $34 \mathrm{GHz}$. These cw ESR measurements were carried out at $50 \mathrm{~K}$ using a field-modulation frequency of $100 \mathrm{kHz}$, modulation amplitude of $0.3-0.7 \mathrm{mT}$, and microwave power of $0.9-7.8 \mathrm{~mW}$.

\section{RESULTS}

\section{A. Spin-lattice relaxation time}

Figure 1(a) shows spin-lattice relaxation curves at $30 \mathrm{~K}$ of the undoped sample $(p=9.1$ at. $\%)$. Solid and open circles were measured at magnetic fields of 389.0 and $391.8 \mathrm{mT}$ where the echo signals originate mainly from $g=2.004$ and 2.01 signals, respectively [see Fig. 1(b)]. These curves were obtained by a pulse sequence of three-pulse inversion recovery $\left(180^{\circ}\right.$ pulse $-t-90^{\circ}$ pulse $-\tau-180^{\circ}$ pulse $-\tau-$ echo, $\tau$ $=240 \mathrm{nsec}, t$ is scanned, RT $=30 \mathrm{msec}) .{ }^{24}$ Solid lines in the figure represent fitted curves with stretched-exponential functions $I(t) / I(0)=1-2 \exp \left[-\left(t / T_{1}\right) \beta\right]$, where $T_{1}$ is the spin-lattice relaxation time and $\beta$ is a dispersion parameter $(0 \leqslant \beta \leqslant 1) .{ }^{24}$ Values of $T_{1}$ and $\beta$ at $30 \mathrm{~K}$ for $g=2.004$ and 2.01 are summarized in Table II. It is found that $T_{1}$ of $g$ $=2.004$ is about three times longer than that of $g=2.01$. This may reflect a difference in electronic structures between conduction- and valence-band-tail spins, which will be dis- 

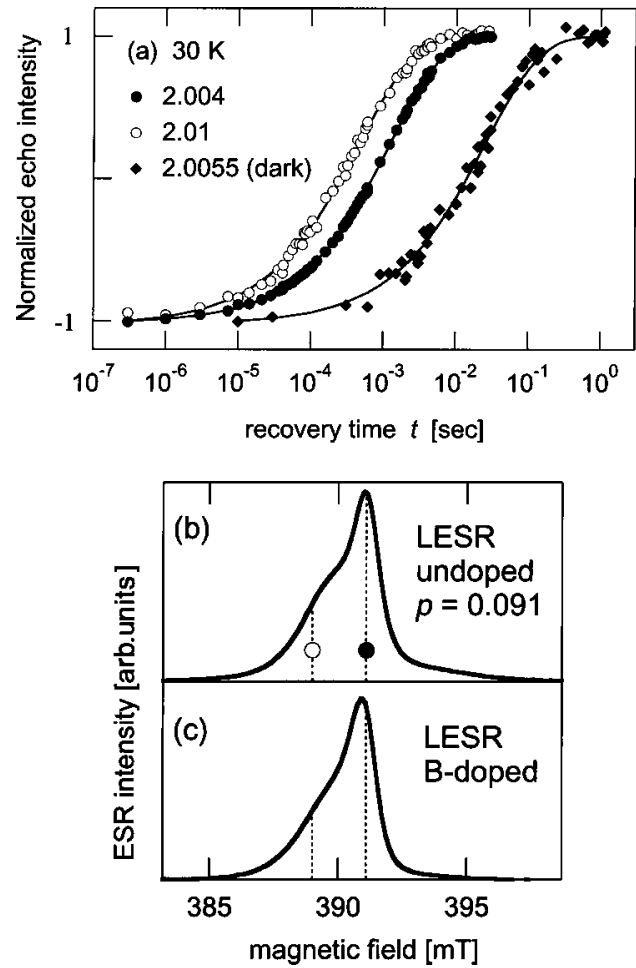

FIG. 1. (a) Echo recovery curves of the $g=2.004,2.01$, and 2.0055 signals at $30 \mathrm{~K}$. Echo intensities are scaled from -1 to 1 . LESR spectra of the (b) undoped and (c) B-doped $a$-Si:H samples. Dashed lines indicate magnetic fields at which we have measured the echo recovery curves. Symbols there correspond to those in $1(\mathrm{a})$.

cussed in Sec. IV A. Such a $T_{1}$ difference was also observed in the conduction- and valence-band-tail spins of $\mathrm{P}$ - and B-doped $a-\mathrm{Si}: \mathrm{H}$ and $a-\mathrm{Ge}: \mathrm{H}$, respectively, although the two centers were separately observed in different samples. ${ }^{26}$

As seen in Fig. 1(a), the dark ESR signal of $g=2.0055$ has a much longer $T_{1}$ than the above two: $T_{1}=26 \mathrm{msec}$. The same relation was also found in illuminated conditions. ${ }^{27}$ Using B-doped $a$-Si:H, we measured the 2.0055 signal and its $T_{1}$ under strong $\left(100 \mathrm{~mW} / \mathrm{cm}^{2}\right)$ illumination [Fig. 1(c)]. This 2.0055 signal is thought to arise from neutral DB's via an electron trapping process at positively charged DB's. The $T_{1}$ value of $g=2.0055$ is six times longer than that of the coexistent 2.01 signal (Table II). Taking advantage of this feature, it is quite possible to reduce the 2.0055 component in

TABLE II. Spin-lattice relaxation parameters of the spin centers. $B_{r}$ represents magnetic fields at which we have measured relaxation curves.

\begin{tabular}{lcccc}
\hline \hline Experimental conditions & $\mathrm{g}$ & $\begin{array}{c}B_{r} \\
(\mathrm{mT})\end{array}$ & $\begin{array}{c}T_{1} \\
(\mathrm{msec})\end{array}$ & $\beta$ \\
\hline \multirow{4}{*}{ undoped, $p=9.1$ at. $\%(30 \mathrm{~K})$} \\
In the dark \\
Under illumination & 2.0055 & 391.1 & 26 & 0.58 \\
& 2.01 & 389.0 & 0.47 & 0.61 \\
& 2.004 & 391.8 & 1.3 & 0.59 \\
Under illumination & B-doped (50 K) & & \\
& 2.01 & 389.0 & 0.06 & 0.59 \\
& 2.0055 & 391.1 & 0.38 & 0.69 \\
\hline \hline
\end{tabular}

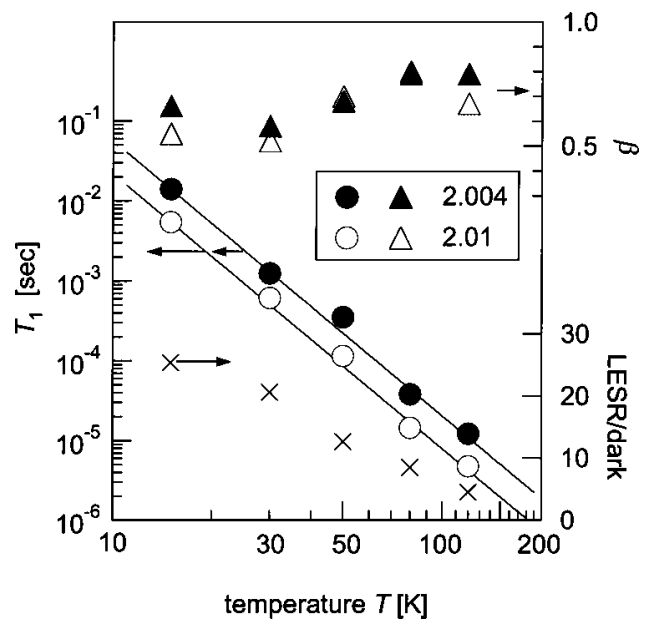

FIG. 2. Temperature dependence of $T_{1}$ (circles) and $\beta$ (triangles). The data were obtained for undoped $a$-Si:H with $p$ $=4.7$ at. $\%$. Solid lines indicate the dependence $T^{-3.4}$. "LESR/ dark' represents the ratio of peak heights of the LESR spectrum to the dark ESR spectrum.

the LESR spectrum. The 2.0055 component should be decreased if we apply RT just long enough for the 2.004 and 2.01 components to recover to the equilibrium states (for example, $10 \mathrm{msec}$ at $30 \mathrm{~K}$ ), because such a RT is not sufficient for full recovery of the longer- $T_{1}$ component, $g$ $=2.0055$.

The dispersion parameter $\beta$ is found in the range from 0.6 to 0.7 for every center (Table II). $\beta$ values smaller than unity indicate that $T_{1}$ of each center has a distribution in the vicinity of the major value listed in Table II. This is reasonable because of the amorphous nature of the material. The distribution of $T_{1}$ values did not affect the echo-detected ESR experiments in the dark, shown by the fact that the shape of the dangling-bond signal was unchanged with varying RT. The shape of the LESR signals, however, was distorted when we adopted a RT much shorter than $T_{1}$. The distortion of the line shape was detected only in the center part of the spectra where $g=2.004$ and 2.01 signals have considerable overlap. Such distortion is predominantly caused by the cross relaxation between the 2.004 and 2.01 spins rather than the $T_{1}$ distributions, because the cross relaxation is more effective in the overlapping region. ${ }^{28}$

The temperature dependence of $T_{1}$ and $\beta$ is shown in Fig. 2. Generally, $T_{1}$ obeys a power-law dependence with respect to temperature $T$, i.e., $T_{1} \propto T^{-m}{ }^{28}$ Actually, $T_{1}$ for both $g$ $=2.004$ and 2.01 obeyed a similar power law with $m \approx 3.4$ from 15 to $120 \mathrm{~K}$, slightly different from $m=2-3$ for doped $a$-Si:H from 30 to $100 \mathrm{~K}^{26}$ The value of 3.4 is difficult to understand from simple models for the spin-lattice relaxation process. ${ }^{28}$ Peak heights of LESR spectra normalized to those of dark ESR spectra are also shown in the figure. Since the LESR intensity is much greater than the dark ESR intensity, the contribution of $g=2.0055$ to $T_{1}$ is not observable.

\section{B. ${ }^{29} \mathrm{Si}$ hyperfine structure of the LESR spectrum}

Figure 3 shows LESR spectra of undoped samples with $p=1.6,4.7$, and 9.1 at. \%, which were obtained by the twopulse Hahn echo sequence with RT of $10 \mathrm{msec}$. Spin densi- 


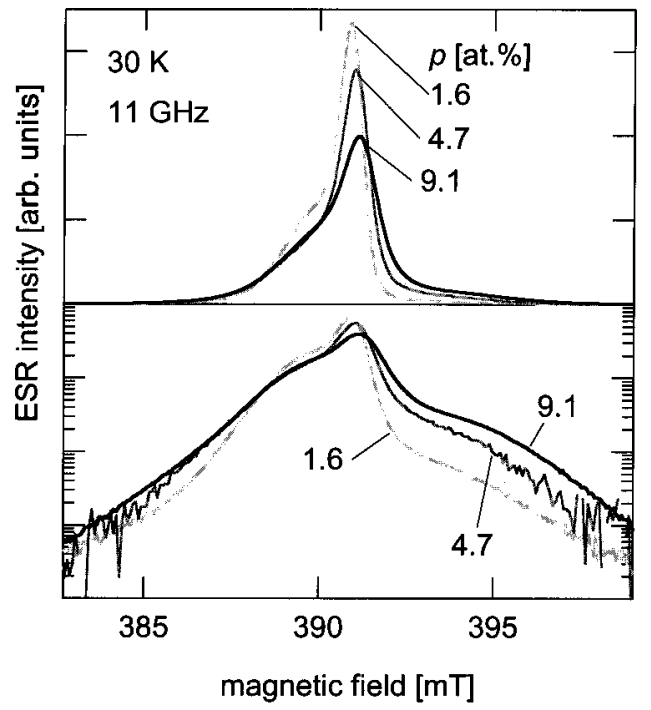

FIG. 3. ${ }^{29} \mathrm{Si}$ concentration dependence of the LESR spectrum of undoped $a-\mathrm{Si}: \mathrm{H}$. Intensities of those spectra are normalized to their areas. The upper and lower figures are linear and logarithmic plots, respectively.

ties of these spectra were found to lie within the range from $8 \times 10^{16}$ to $1.0 \times 10^{17} \mathrm{~cm}^{-3}$, more than 20 times larger than those of the dark ESR signals obtained by the same pulse sequence. When the light intensity is sufficiently high, as in the present case, LESR spectra no longer depend on the relative intensity between the LESR and the dark ESR signals, although under very weak illumination (or at relatively high temperatures) LESR spectra seem to be influenced by the dark ESR component. ${ }^{29,30}$ This means that there is no substantial contribution of the dark ESR component to the observed LESR spectrum for the strong-illumination cases.

With increasing ${ }^{29} \mathrm{Si}$ concentration, it was found that the peak height of $g=2.004$ was reduced considerably. This is due to a signal broadening of the $g=2.004$ signal induced by unresolved ${ }^{29} \mathrm{Si}$ hf splittings, which is effective for the $g$ $=2.004$ signal because of its narrow linewidth. An apparent shift in the peak position of the 2.004 signal is also a consequence of the reduction in the peak height of $g=2.004$ relative to $g=2.01$. The ${ }^{29} \mathrm{Si}$ concentration dependence shown here indicates that excessive doping of ${ }^{29} \mathrm{Si}$ in the film causes extreme signal broadening, which smears out the separation between the 2.004 and 2.01 signals as well as ${ }^{29} \mathrm{Si}$ hf splittings.

As seen in the logarithmic plot of Fig. 3, there is a weak and broad signal in the tail region of every LESR spectrum. These signals are high-magnetic-field-side ${ }^{29} \mathrm{Si}$ hf lines, because the area ratio $S_{\text {high-hf }}$, of these lines to the total spectrum increased proportionally to $p$, as seen in Fig. 4(a). The deconvolution of the high-field-side hf lines was performed by extrapolating a tail curve from the main peak of $g$ $=2.004$, in which we assumed an exponential decay of the tail. ${ }^{22}$ Likewise, in Fig. $4(\mathrm{~b})$, the $S_{\text {high-hf }}$ values are estimated for the dangling-bond signal of $g=2.0055$. Solid lines represent the relation $S_{\text {high-hf }}=p / 2$, which corresponds to the fact that the wave function of the neutral DB center is localized mainly on a single $\mathrm{Si}$ atom. ${ }^{12-14}$ A good coincidence between the line and plots in Fig. 4(b) assures the validity of the deconvolution of high-field hf lines. For the LESR spec-

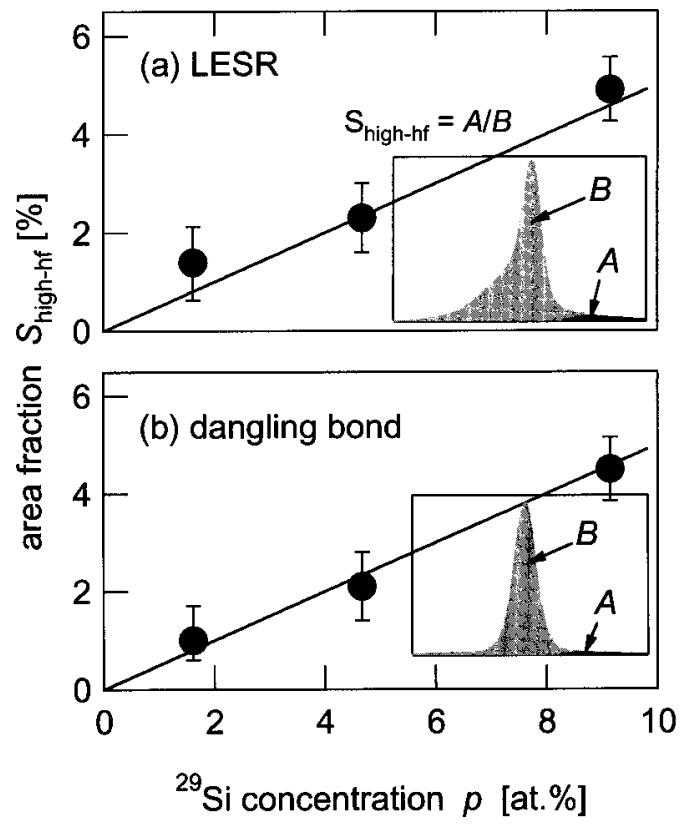

FIG. 4. ${ }^{29} \mathrm{Si}$ concentration dependence of the area fraction ( $S_{\text {high-hf }}$ ) of the high-field-side ${ }^{29} \mathrm{Si} \mathrm{hf}$ line: (a) for LESR and (b) for dark ESR spectra. $S_{\text {high-hf }}$ is given by the area ratio $A / B$.

trum, $S_{\text {high-hf }}$ seems to trace a similar line as in the danglingbond case. Previously, Yamasaki et al. ${ }^{19}$ tentatively suggested that the wave function of the LESR spin centers is localized on a single Si atom, simply because the value of $S_{\text {high-hf }}$ was close to $p / 2$ for $p=4.7$ at. \% [see Fig. 4(a)]. However, the data are not sufficient to discuss, because the LESR spectrum is composed of two signals $(g=2.004$ and $2.01)$. In order to obtain more detailed and correct information, it is necessary to deconvolute the LESR spectrum into the two signals. Fortunately, by taking advantage of the difference in $T_{1}$ between the two LESR centers (see Sec. III A), it is quite possible to experimentally deconvolute the LESR spectrum, as we reported previously. ${ }^{22}$

Figure 5 shows the result of the experimental deconvolution for $p=4.7$ at. \% (a natural-abundance sample). The "total" spectra in Figs. 5(a) and 5(b) were measured by echodetected ESR techniques using a two-pulse Hahn echo (RT = $0.5 \mathrm{msec}$ ) and a three-pulse stimulated echo $\left(90^{\circ}\right.$ pulse $-\tau-90^{\circ}$ pulse $-\tau^{\prime}-90^{\circ}$ pulse $-\tau-$ echo, $\quad \tau=240$ nsec, $\tau^{\prime}=1 \mathrm{msec}, \mathrm{RT}=10 \mathrm{msec}$ ), respectively. $\tau^{\prime}$ longer than $1 \mathrm{msec}$ was not effective because of a serious reduction of the echo intensity. The details of the deconvolution procedure have been reported in Ref. 22. The center part of the LESR spectra could not be deconvoluted completely because of the distortion of the spectra as discussed in the preceding section. Figure 5 clearly demonstrates that the ${ }^{29} \mathrm{Si}$ highfield-side hf line of the LESR spectrum is related mainly to the 2.004 signal, which is the most important feature. Peak positions of the two deconvoluted signals were found to correspond to $g=2.004$ and 2.01, respectively. The area ratio of the deconvoluted two signals was estimated to be [2.004]:[2.01] $\approx 1.3: 1$ for the normal LESR spectrum shown in Fig. 3. The same results were also obtained for a ${ }^{29}$ Si-enriched sample $(p=9.1$ at. $\%) .{ }^{22}$ For a ${ }^{29}$ Si-diluted sample ( $p=1.6$ at. \%), it was rather difficult to get sufficient intensity of the ${ }^{29} \mathrm{Si}$ structure after the deconvolution process. 


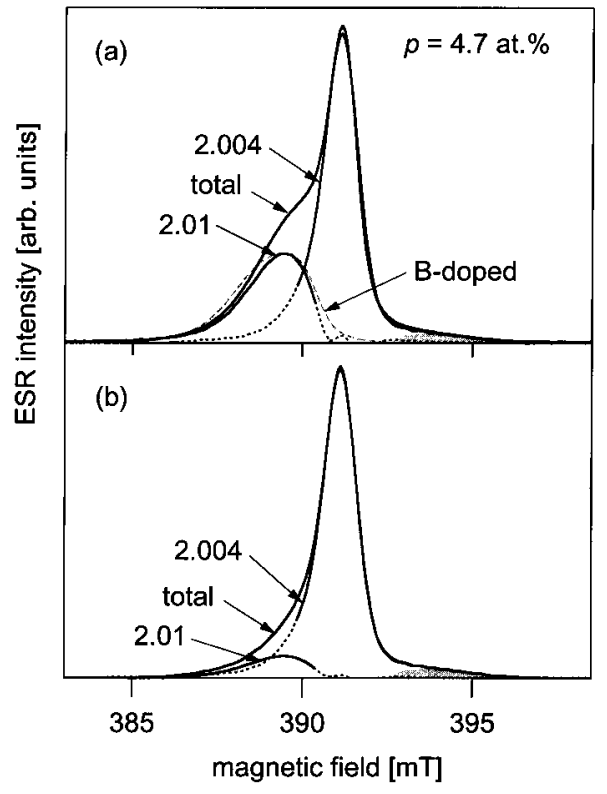

FIG. 5. Experimental deconvolution of two LESR signals for the case of $p=4.7$ at. \%. Solid lines indicate the deconvoluted 2.004 and 2.01 spectra with high reliability in data analyses, while dashed lines include some ambiguity. Shaded regions show the high-field-side hf structure of ${ }^{29} \mathrm{Si}$. The dark ESR spectrum of B-doped $a-\mathrm{Si}: \mathrm{H}$ is also shown by a broken line.

\section{Electronic structure of LESR centers}

In this section, we extract detailed information about the electronic structures of LESR centers from the deconvoluted ${ }^{29} \mathrm{Si}$ hf structures. One of the most important pieces of information is the area fraction $S_{\mathrm{hf}}$ of the entire ${ }^{29} \mathrm{Si}$ hf structure (a group of low- and high-field-side hf lines) in the deconvoluted signal. The quantity $S_{\mathrm{hf}}$ is determined by the number of $\mathrm{Si}$ atoms, $N$, on which an electron spin is mainly located. When $N=1, S_{\text {hf }}$ equals simply $p / 2+p / 2=p$, as is illustrated in Fig. 6(a). For any $N$, since the probability that all $N \mathrm{Si}$ atoms have no nuclear spin is $(1-p)^{N}, S_{\mathrm{hf}}$ should be given by $1-(1-p)^{N}$. Although this includes the small probability of ${ }^{29} \mathrm{Si}$ hf lines overlapping on a central line, e.g., a probability of $p^{2} / 2$ for the $N=2$ case shown in Fig. 6(b), such a small probability is negligible for the case of small $N$ and $p$. The value of $S_{\mathrm{hf}}$ is experimentally determined by the area ratio $A / B$ of the deconvoluted signal, as shown in Fig. 6(c). The area $B$ is calculated using the high-field-side half of the deconvoluted 2.004 spectrum in order to eliminate an ambiguity in the "dashed-line" region (cf. Fig. 5). The high-fieldside ${ }^{29} \mathrm{Si}$ hf structure was estimated by the procedure used in Sec. III B. As a result, the $S_{\mathrm{hf}}$ values were estimated to be $0.20 \pm 0.03$ and $0.09 \pm 0.03$ for $p=0.091$ and 0.047 , respectively. Thus, for the 2.004 center,

$$
N= \begin{cases}2.3 \pm 0.4 & \text { for } p=0.091 \\ 2.1 \pm 0.4 & \text { for } p=0.047\end{cases}
$$

Consequently, it is concluded that the wave function of the 2.004 center spreads mainly over two $\mathrm{Si}$ atoms. ${ }^{22}$ In the above discussion, we assumed that the wave function of the spin center spreads over $N \mathrm{Si}$ atoms uniformly, although the actual wave function may fluctuate among $N \mathrm{Si}$ atoms. A more exact analysis needs to consider variations of ${ }^{29} \mathrm{Si} \mathrm{hf}$

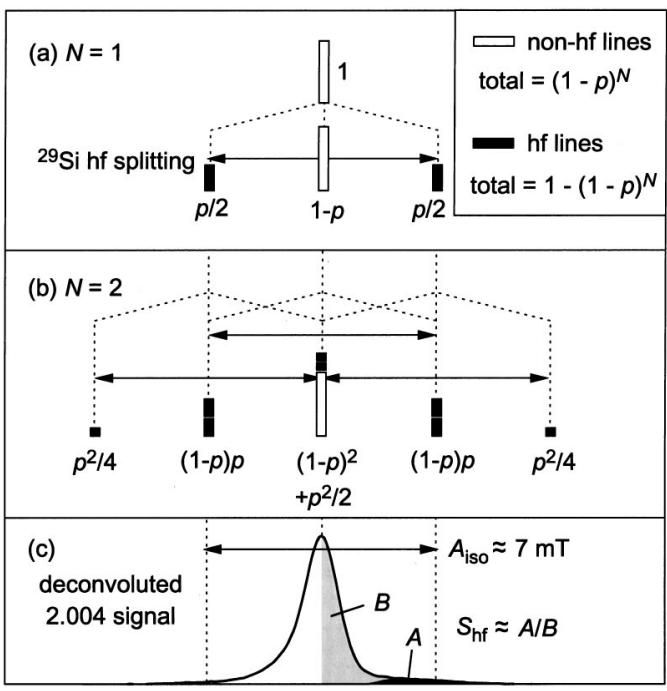

FIG. 6. Schematic views of ${ }^{29} \mathrm{Si}$ hf splitting for cases of (a) $\mathrm{N}$ $=1$ and (b) $N=2$, assuming two equivalent hf splittings. (c) Estimation of $S_{\mathrm{hf}}$ and $A_{\text {iso }}$ for the deconvoluted 2.004 signal.

interactions among $N \mathrm{Si}$ atoms. However, the fluctuation contributes only to the linewidth of the hf structure and therefore does not affect the above conclusion.

Another interesting feature is the isotropic hf splitting $A_{\text {iso }}$, which arises mainly from the $3 s$ character in the wave function of a spin center. ${ }^{12-14}$ As is shown in Fig. 6(c), the isotropic hf splitting is estimated to be

$$
A_{\text {iso }} \approx 7 \mathrm{mT} \text { for } g=2.004 \text {. }
$$

In contrast to the 2.004 signal, the 2.01 signal did not appear to show hf structure. Therefore, the isotropic hf splitting of $g=2.01$ should be smaller than that of $g=2.004$. The 2.01 signal in B-doped $a$-Si:H [Fig. 5(a)] also shows no discernible hf structures. ${ }^{21}$ Furthermore, both the 2.01 signals are similar in their asymmetric line shape. The origin of the asymmetric line shape of $g=2.01$ is ascribed either to the powder pattern ${ }^{31}$ due to anisotropy of the $g$ value, or to the convolution of hf structures. To clarify the origin, ESR spectra of $g=2.01$ were measured at microwave frequencies $(\nu)$ of 3,11,34 GHz, and are shown in Fig. 7(a). In the figure, although the linewidth was reduced by less than $1.0 \mathrm{mT}$ in the $3 \mathrm{GHz}$ spectrum, distortion of the spectral shape due to isotropic hf interactions was still not observed. This means that the isotropic hf splittings of $g=2.01$ are smaller than 3 $\mathrm{mT}$, i.e.,

$$
A_{\text {iso }}<3 \mathrm{mT} \text { for } g=2.01 \text {, }
$$

and hence the asymmetric line shape of $g=2.01$ originates from the powder pattern. In Fig. 7(b), ESR intensities are plotted as a function of $g$ value. The asymmetry in the line shape is enhanced in the $34 \mathrm{GHz}$ spectrum in Fig. 7(b), which clearly indicates a convolution of the powder pattern. To estimate principal $g$ values of the 2.01 signal, we carried out a powder-pattern simulation of the 2.01 spectra. In the simulation, an ideal powder pattern ${ }^{31}$ was broadened by convoluting the Voigt broadening function with the full width at half maximum, $W$, and a shape parameter $y .{ }^{14} \mathrm{We}$ assumed 

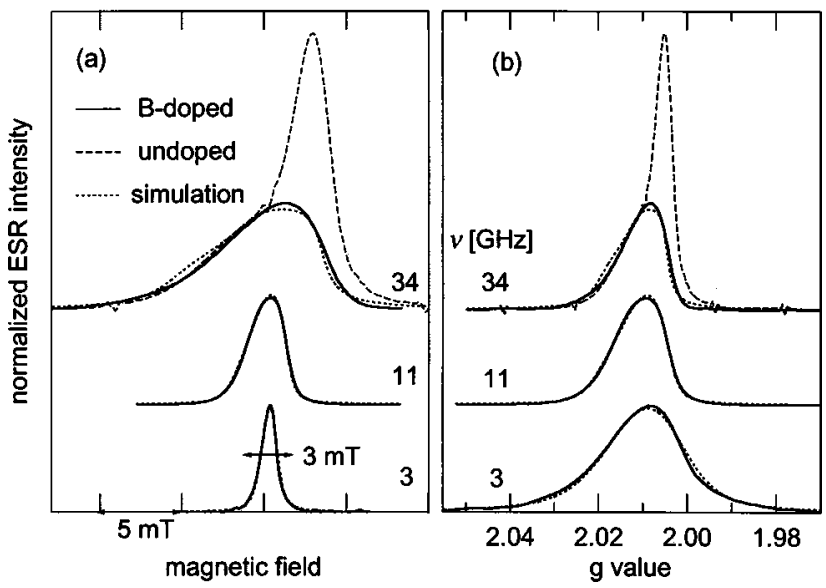

FIG. 7. Multifrequency $(3,11,34 \mathrm{GHz})$ ESR measurements on the $g=2.01$ signal in B-doped $a-\mathrm{Si}: \mathrm{H}$ (solid lines). ESR intensities are plotted (a) as a function of applied magnetic field $B$, and (b) as a function of $g$ value, where $g=[71.4488 \nu(\mathrm{GHz})] /[B(\mathrm{mT})]$. The 2.01 spectra are normalized to their peak heights and are vertically shifted for clarity. Dashed lines indicate simulated powderpattern spectra. LESR spectra in undoped $a-\mathrm{Si}: \mathrm{H}(p=9.1$ at. \%) for $34 \mathrm{GHz}$ are also shown by broken lines, with intensities modified to fit their 2.01 signals approximately to the B-doped 2.01 signal.

that the width $W$ is proportional to the $g$ shift $(\Delta g)$ from the free-electron $g$ value (2.0023) as well as the resonant frequency, because such a dependence has been observed in the dangling-bond spectrum in $a$-Si:H. ${ }^{14}$ The fitting parameters were $g_{1}, g_{2}$, and $g_{3}$ (principal $g$ values $g_{1}>g_{2}>g_{3}$ ), $W_{0}$ and $W_{1}$ (width parameters: $W \equiv W_{0}+W_{1} \Delta g \nu$ ), $C$ (normalizing constant), and $y$, where $g_{1}, g_{2}, g_{3}, W_{0}$, and $W_{1}$ were set to be common for all the spectra. The simulation minimized the sum of deviations among three experimental and fitted spectra using the same algorithm as in previous work. ${ }^{14}$ The best-fitted spectra (dashed lines in Fig. 7) were obtained for $g_{1} \approx 2.019, g_{2} \approx 2.012$, and $g_{3} \approx 2.005$. One of the $g$ values $\left(g_{3}\right)$ was close to $g=2.004$, so the two LESR signals of $g$ $=2.004$ and 2.01 were not completely separated even in the $34 \mathrm{GHz}$ spectrum, as shown in Fig. 7.

It has been reported that the energetic positions in the gap are deeper for valence-band-tail holes than for conductionband-tail electrons, resulting in stronger localization for $g$ $=2.01$ centers than $g=2.004$ ones. ${ }^{3,8,32}$ The stronger localization must cause larger isotropic splitting, unless the wave function of the spin center has less $3 s$ component. Thus, the weaker isotropic hf interaction of $g=2.01$ means that the wave function of the 2.01 center has much less $3 \mathrm{~s}$ component compared to the 2.004 center; in other words, it consists of $3 p$ component predominantly. This difference will be interpreted later in terms of the origin of the LESR centers. Although the 2.01 center has much $3 p$ component, it is difficult to detect the anisotropic ${ }^{29} \mathrm{Si}$ hf interactions in the 2.01 spectrum, because a $100 \%$ localized $3 p$ orbital of the ${ }^{29} \mathrm{Si}$ atom causes an anisotropic hf splitting of no more than 3.6 $\mathrm{mT} \cdot{ }^{33}$

The electronic levels that are located in the vicinity of the mobility gap of $a-\mathrm{Si}: \mathrm{H}$ are characterized by a combination of $3 s$ and $3 p$ levels of $\mathrm{Si}$ atoms. Thus, to consider the electronic structures of band-tail states, it is useful to describe the wave function $\psi$ of $g=2.004$ and 2.01 by a linear combination of atomic orbitals. That is $\psi=\sum_{i} \alpha_{i}\left(\sigma_{i}|3 s\rangle+\pi_{i}|3 p\rangle\right)$, where $|3 s\rangle$ and $|3 p\rangle$ denote the atomic $3 s$ and $3 p$ orbitals of $\mathrm{Si}, i$ indexes all $\mathrm{Si}$ atoms within the extent of $\psi, \alpha_{i}^{2}$ represents the degree of localization of an unpaired electron on site $i$, and $\sigma_{i}^{2}$ and $\pi_{i}^{2}$ are fractions of $3 s$ and $3 p$ orbitals on site $i$, respectively. ${ }^{12,14}$ The projection coefficients satisfy the normalization conditions $\Sigma_{i} \alpha_{i}^{2}=1$ and $\sigma_{i}^{2}+\pi_{i}^{2}=1$ for all $i$. The isotropic hf splitting $A_{\text {iso }}$ has been theoretically calculated to be $149.0 \mathrm{mT}$ for a $100 \%$ localized $3 s$ orbital of ${ }^{29} \mathrm{Si}$ atom. ${ }^{33}$ Comparing Eq. (2) with this value, the $3 s$ component in the wave function of the 2.004 center is estimated to be $7 / 149=5 \%$ on each of the two Si atoms. On all other $i$ sites, the $3 \mathrm{~s}$ components should be much smaller than $5 \%$. Therefore,

$$
\begin{gathered}
\alpha_{1}^{2} \sigma_{1}^{2} \approx \alpha_{2}^{2} \sigma_{2}^{2} \approx 0.05, \\
\alpha_{i}^{2} \sigma_{i}^{2}<0.05 \quad(i \neq 1,2)
\end{gathered}
$$

for $g=2.004$, where $i=1$ and 2 represent the two $\mathrm{Si}$ atoms counted in $N$. For the 2.01 center, all $\alpha_{i}^{2} \sigma_{i}^{2}$ should be smaller than $3 / 149=2 \%$, because $A_{\text {iso }}<3 \mathrm{mT}$ [Eq. (3)], namely,

$$
\alpha_{1}^{2} \sigma_{1}^{2}<0.02 \text { for all } i
$$

for $g=2.01$. The localization coefficients $\alpha_{1}^{2}$ and $\alpha_{2}^{2}$ for $g$ $=2.004$ should be larger than those for $g=2.01$, as we mentioned above. Thus, to satisfy Eqs. (4) and (5), $\sigma_{1}^{2}$ and $\sigma_{2}^{2}$ for $g=2.01$ should be much smaller than those for $g=2.004$,

$$
\left.\sigma_{1}^{2}, \sigma_{2}^{2} \quad(\text { for } g=2.01) \ll \sigma_{1}^{2}, \sigma_{2}^{2} \text { (for } g=2.004\right) .
$$

A further evaluation of the projection coefficients has been reported in Ref. 22.

\section{DISCUSSION}

\section{A. Origin of LESR centers}

For the origin of the LESR centers, two models have so far been proposed. In this section, those models are compared with the ESR results obtained here, in particular, with Eqs. (1) and (6).

One model is that of electrons or holes trapped at weak $\mathrm{Si}-\mathrm{Si}$ bonds. ${ }^{34} \mathrm{In}$ an amorphous network, it is quite possible that greatly elongated covalent bonds (weak bonds) are present and give rise to the energy levels in the band-tail regions. In this case, an electron is trapped in an antibonding state and its wave function extends over the two $\mathrm{Si}$ atoms associated with the weak Si-Si bond. In the other model, electrons and holes are trapped at positively and negatively charged DB's with negative $U$, resulting in neutral DB's with $g=2.004$ and 2.01, respectively. ${ }^{17-20}$ The resultant neutral DB's are thought to be similar to those observed in the dark. However, a neutral DB state is characterized by an electron localized on a single $\mathrm{Si}$ atom (namely, $N \approx 1$ ), ${ }^{12-14}$ which is clearly inconsistent with the present result of $N$ $\approx 2$ [Eq. (1)]. Consequently, the present results suggest that the origin of the LESR center of $g=2.004$ is electrons trapped at weak Si-Si bonds located at the conduction-bandtail rather than electrons trapped at positively charged DB's. 
It is reasonable to consider for the $g=2.01$ center, by analogy with the 2.004 center, that it originates from holes trapped in the bonding states of the weak Si-Si bonds located in the valence-band tail. The weak Si-Si bond model can explain why the 2.01 center has less $3 s$ component in its wave function than the 2.004 center [cf. Eq. (6)]. As in crystalline silicon, the top of the valence band and the bottom of the conduction band in $a-\mathrm{Si}: \mathrm{H}$ are characterized by $3 p$ orbital ( $p \sigma$ state) and an admixture of $3 s$ and $3 p$ orbitals $\left(s p \sigma^{*}\right.$ state), respectively. ${ }^{35}$ The antibonding and bonding states of the weak Si-Si bond retain essentially similar features to normal Si-Si bonds. Therefore, the wave function of holes trapped in the bonding states of the weak bonds will consist of much less $3 s$ component than that of electrons in the antibonding levels, resulting in smaller isotropic hf splitting of $g=2.01$.

The above discussion is supported by more quantitative evaluations. Takeda et al. reported a first-principles localdensity-functional calculation of a weak bond with $27 \%$ stretching embedded in polysilane chains $\left(\mathrm{Si}_{4} \mathrm{H}_{8}\right.$ and $\left.\mathrm{Si}_{6} \mathrm{H}_{12}\right){ }^{36}$ They showed that holes localize at the center of the weak bond and the hole wave function is composed mainly of $3 p$ orbital, while electrons tend to localize at two $\mathrm{Si}$ atoms of the weak bond and its wave function has a $3 \mathrm{~s}$ component. As a result, the isotropic hf splitting of the hole center was calculated to be less than $\frac{1}{70}$ of that of the electron center. The energy separation between the two weak-bond levels was estimated to be $65 \%$ of the energy gap for polysilane chains $(=3.9 \mathrm{eV})$, which is comparable with the actual range between $60 \%$ and $100 \%$ of $1.7 \mathrm{eV}$ in $a-\mathrm{Si}: \mathrm{H} .{ }^{1,2,8}$ More recently, Ishii and Shimizu calculated ${ }^{29} \mathrm{Si}$ hf interactions in negatively and positively charged weak bonds with 50-75\% stretching by means of the density-functional method for clusters with 8-26 Si atoms. ${ }^{37}$ They found that the isotropic hf splitting for the negatively charged weak bond becomes $6-11 \mathrm{mT}$ as against $0.1-0.2 \mathrm{mT}$ for the positively charged one, which is quite consistent with our experimental results.

Note that any $s$ orbitals make little contribution to the spin-orbit interaction, because their orbital angular momentum is zero. ${ }^{38}$ Thus, due to more $3 s$ component in the wave function of $g=2.004$, the 2.004 center tends to generate less spin-orbit coupling, resulting in a longer $T_{1}$ and smaller $g$ shift compared to the 2.01 center. Such a trend is consistent with the $T_{1}$ difference and the $g$ shift observed in the experiment.

In contrast to the weak bond, charged DB's are unlikely to adopt different isotropic hf splittings between $g=2.004$ and 2.01. As is seen in Fig. 1(c) for the case of positively charged DB's, both positively and negatively charged DB's are probably converted to neutral DB's with isotropic hf splitting close to $7 \mathrm{mT}$.

A detailed study of the electron-spin-echo envelope modulation revealed that the LESR centers are spatially separated from hydrogen atoms by approximately $0.4 \mathrm{~nm} .{ }^{25}$ This is quite reasonable for the weak Si-Si bond model, because insertion of hydrogen in a disordered Si network may relax local stress and prevent the formation of elongated covalent $\mathrm{Si}-\mathrm{Si}$ bonds near hydrogen atoms. Conventional $a$-Si:H films contain other impurities such as oxygen, carbon, and nitrogen with concentrations of $10^{18}-10^{20} \mathrm{~cm}^{-3} .^{39}$ However, a high-purity undoped film, which contains no
TABLE III. Comparison of data for conduction-band-tail electrons from the present LESR and the previous PL and ODMR (Refs. 40-42, 44) experiments.

\begin{tabular}{lcc}
\hline \hline & LESR & PL and ODMR \\
\hline Spatial extent & smaller than $1 \mathrm{~nm}$ & $\approx 1 \mathrm{~nm}$ \\
Time constant of decay & $>10^{2} \mathrm{sec}$ & $\approx 10^{-3} \mathrm{sec}$ \\
g value & 2.004 & $\approx 1.99$ \\
Concentration & $\leqslant 10^{17} \mathrm{~cm}^{-3}$ & $\geqslant 10^{18} \mathrm{~cm}^{-3}$ \\
Energy positions & deep & shallow \\
Origin of trap center & weak Si-Si bond & $?$ \\
\hline \hline
\end{tabular}

more than $10^{16} \mathrm{~cm}^{-3}$ of impurities, still reveals the band-tail structure in its optical absorption spectrum. ${ }^{39}$ Therefore, we speculate that the impurities do not directly relate to the formation of weak bonds, and thus the weak bond may be an intrinsic structure of the amorphous silicon network.

\section{B. Comparison between LESR centers and other band-tail states}

Our experiment revealed that the $g=2.004$ electrons generate only two main isotropic hf interactions of ${ }^{29} \mathrm{Si}$. On the other hand, previous photoluminescence (PL) and spindependent PL [the so-called optically detected magnetic resonance (ODMR)] experiments suggested that the spatial extent of the conduction-band-tail electrons is as large as 1 $\mathrm{nm} .{ }^{40,41}$ Those experiments analyzed the PL signal at a photon energy of 1.2-1.4 eV which has been attributed to the radiative recombination between conduction-band-tail electrons and valence-band-tail holes. ${ }^{7,40,42}$ Normally, a sphere of $1 \mathrm{~nm}$ radius in $a$-Si:H ( $\mathrm{Si}$ density $\approx 5 \times 10^{22}$ atoms $/ \mathrm{cm}^{-3}$ ) contains more than $150 \mathrm{Si}$ atoms. ${ }^{43}$ Thus, the PL electrons are considered to have substantial electron densities on several dozen $\mathrm{Si}$ atoms and therefore do not adopt the same ${ }^{29} \mathrm{Si}$ hf interactions as the 2.004 electrons.

In order to interpret the difference between the LESR and PL data, we considered that the band-tail electrons observed in the PL possess relatively higher energetic positions and larger spatial extent compared to the 2.004 electrons in LESR. This speculation is consistent with various experimental facts, which are summarized in Table III. (1) The time constant of the decay is quite different between the two experiments; below $50 \mathrm{~K}$, the PL intensity decays with a time constant of $10^{-3} \mathrm{sec}$ after excitation, ${ }^{40}$ but for the LESR intensity, the constant is larger than $10^{2} \mathrm{sec}$. The much slower decay for the LESR may be ascribed to the deeper energy location of the 2.004 electrons. Since the PL intensity decreases proportionally with the rate of radiative recombination, it is quite difficult for PL to detect the 2.004 electrons, which have a very long recombination lifetime. (2) The $g$ value is quite different; a time-resolved ODMR measurement under intense pulsed excitations (peak power $\approx 220 \mathrm{~kW} / \mathrm{cm}^{2}$ ) detected a very weak signal of $g \approx 1.99$ which was tentatively ascribed to band-tail electrons, ${ }^{44}$ but such a signal is similar to a conduction-electron resonance in microcrystalline silicon ${ }^{45}(g=1.998)$ rather than $g=2.004$. This suggests that the PL electrons are located in shallow levels close to the mobility edge. (3) The carrier concentration is different; the concentration of the PL electrons is eas- 
ily higher than $10^{18} \mathrm{~cm}^{-3},{ }^{40}$ while that of the 2.004 electrons did not exceed $10^{17} \mathrm{~cm}^{-3} .{ }^{30}$ It also leads to higher-energy positions for the PL electrons. Further, this fact suggests that the 2.004 centers relate to only a part of the conductionband-tail states, although the PL electrons may be responsible for the major part.

The reason that LESR and PL revealed different conduction-band-tail electrons is tentatively ascribed to the following features of the LESR. The LESR measurements are efficient in detecting band-tail electrons having a long recombination lifetime $\left(\tau_{r}\right)$, because the steady-state density $N$ of band-tail electrons should increase as $\tau_{r}$ increases (note that the steady-state rate equation $d N / d t=G-N / \tau_{r}=0$, where $G$ is the generation rate of band-tail electrons, results in $N=G \tau_{r}$ ). In addition, it is also required that unpaired spins have a $T_{1}$ long enough to avoid a serious reduction in the ESR signal intensity due to lifetime broadening. Naturally, localized electrons in deeper band-tail levels have longer $\tau_{r}$ (and may also have longer $T_{1}$ ) as compared to delocalized electrons in shallower band-tail levels. Therefore, LESR will be increasingly effective for deeper band-tail levels, resulting in smaller spatial extent for the LESR electrons than for the PL ones.

As mentioned earlier, the ${ }^{29} \mathrm{Sihf}$ structure of the PL electrons may be different from that of the LESR electrons. For example, discernible ${ }^{29} \mathrm{Si}$ hf splitting might be absent in the ODMR spectrum due to the delocalization of unpaired electrons. Unfortunately, previous experimental results as well as the present work are not sufficient to determine what sort of structural fluctuation causes such weakly localized centers, with a spatial extent of $1 \mathrm{~nm}$, having a density of states higher than $10^{18} \mathrm{~cm}^{-3}$. For the weak-bond center, theoretical calculations suggest $0.3-0.4 \mathrm{~nm}$ for the $\mathrm{Si}-\mathrm{Si}$ bond length. ${ }^{36,37,46}$ Weak $\mathrm{Si}-\mathrm{Si}$ bonds whose $\mathrm{Si}$-Si bond length is closer to a normal Si-Si length $(0.235 \mathrm{~nm})$ than to 0.3 to 0.4 $\mathrm{nm}$ might be one of the origins of the weakly localized centers. But even so, many kinds of structural fluctuation will contribute to the formation of the centers because of their large spatial extent. For example, it was reported that fluctuations of either bond-angle or ring statistics rather than bond length are effective in generating band-tail states. ${ }^{47}$ Thus, the nature of the weakly localized centers is probably different from that of the 2.004 center.

\section{SUMMARY}

This paper follows up our previous work ${ }^{22}$ with more complete experimental data and discussion. We have inves- tigated the electronic and microscopic structures of the LESR centers of $g=2.004$ (conduction-band-tail electrons) and 2.01 (valence-band-tail holes) in undoped $a-\mathrm{Si}: \mathrm{H}$ on the basis of detailed analyses of their ${ }^{29} \mathrm{Sihf}$ structures. In this study, we took advantage of pulsed ESR techniques, samples with different content of ${ }^{29} \mathrm{Si}$ (1.6, 4.7, 9.1 at. \%), and multifrequency (3-34 GHz) ESR studies, and obtained experimental results as follows.

(1) The $T_{1}$ of the $g=2.004$ center is about three times longer than that of $g=2.01$ (Sec. III A). Using the $T_{1}$ difference, the LESR spectra can be experimentally deconvoluted into two independent signals for $g=2.004$ and 2.01 (Sec. III B).

(2) The fractional intensity of the ${ }^{29} \mathrm{Sihf}$ structure in the deconvoluted signal of $g=2.004$ revealed that the wave function of the 2.004 center spreads mainly over two Si atoms (Sec. IIIC). This suggests that the origin of the 2.004 center is electrons trapped in the antibonding states of the weak $\mathrm{Si}-\mathrm{Si}$ bond rather than those trapped in the positively charged DB's (Sec. IV A). By analogy, we suggested that the 2.01 center arises from holes trapped in the bonding states of the weak $\mathrm{Si}-\mathrm{Si}$ bond.

(3) The ${ }^{29} \mathrm{Si}$ isotropic hf splitting of $g=2.004$ was estimated to be around $7 \mathrm{mT}$, while that of $g=2.01$ was below 3 $\mathrm{mT}$ (Sec. IIIC). This indicates that the wave function of $g$ $=2.01$ consists mainly of the $3 p$ component, while the wave function of $g=2.004$ has more $3 s$ component compared to the 2.01 center. The difference can be accounted for within the framework of the weak-bond model (Sec. IV A).

(4) The anisotropic line shape of $g=2.01$ originates from a large anisotropy of the $g$ value (Sec. III C).

(5) We discussed the difference in the nature of band-tail electrons observed in LESR and PL experiments on the basis of our results and other experimental findings (Sec. IV B). The discussion pointed out that the 2.004 electrons are presumably more localized and are located in deeper band-tail levels than the PL electrons.

\section{ACKNOWLEDGMENTS}

The authors thank Dr. A. Matsuda, Dr. H. Okushi, Dr. H. Katagiri, and Dr. N. Ishii for valuable suggestions, and we are also grateful to Dr. S. Hayashi for NMR analyses of our samples. This work, partly supported by NEDO, was performed in the Joint Research Center for Atom Technology (JRCAT) under the joint research agreement between the National Institute for Advanced Interdisciplinary Research (NAIR) and the Angstrom Technology Partnership (ATP).
*Present address: System Devices and Fundamental Research, NEC Corporation, Tsukuba, 305-8501, Japan.

${ }^{1}$ S. Yamasaki, Philos. Mag. B 56, 79 (1987).

${ }^{2}$ S. Aljishi, J. D. Cohen, S. Jin, and L. Ley, Phys. Rev. Lett. 64, 2811 (1990).

${ }^{3}$ T. Tiedje, J. M. Cebulka, D. L. Morel, and B. Abels, Phys. Rev. Lett. 46, 1425 (1981); W. E. Spear and C. S. Cloude, Philos. Mag. B 58, 467 (1988); M. Kemp and M. Silver, ibid. 63, 437 (1991).

${ }^{4}$ K. Hattori, H. Okamoto, and Y. Hamakawa, J. Non-Cryst. Solids 198-200, 288 (1996).
${ }^{5}$ J. C. Knights, D. K. Biegelsen, and I. Solomon, Solid State Commun. 22, 133 (1977).

${ }^{6}$ H. Dersh, J. Stuke, and J. Bachelor, Phys. Status Solidi B 105, 265 (1981).

${ }^{7}$ R. A. Street, D. K. Biegelsen, and R. L. Weisfield, Phys. Rev. B 30, 5861 (1984).

${ }^{8}$ M. Stutzmann, D. K. Biegelsen, and R. A. Street, Phys. Rev. B 35, 5666 (1987).

${ }^{9}$ S. G. Bishop, U. Strom, and P. C. Taylor, Phys. Rev. Lett. 34, 1346 (1975); 36, 543 (1976).

${ }^{10}$ R. A. Street and N. F. Mott, Phys. Rev. Lett. 35, 1293 (1975). 
${ }^{11}$ M. Kastner, D. Adler, and H. Fritzsche, Phys. Rev. Lett. 37, 1504 (1976).

${ }^{12}$ M. Stutzmann and D. K. Biegelsen, Phys. Rev. B 40, 9834 (1989); in Amorphous Silicon and Related Materials, edited by H. Fritzsche (World Scientific, Singapore, 1988), pp. 557-594.

${ }^{13}$ N. Ishii and T. Shimizu, Phys. Rev. B 42, 9697 (1990).

${ }^{14}$ T. Umeda, S. Yamasaki, J. Isoya, and K. Tanaka, Phys. Rev. B 59, 4849 (1999).

${ }^{15}$ J. D. Cohen, J. P. Harbison, and K. W. Wecht, Phys. Rev. Lett. 48, 109 (1982).

${ }^{16}$ H. Okamoto and Y. Hamakawa, Solid State Commun. 24, 1197 (1977).

${ }^{17}$ D. Adler, Sol. Cells 9, 133 (1983).

${ }^{18}$ K. Morigaki, J. Phys. Soc. Jpn. 50, 2279 (1981).

${ }^{19}$ S. Yamasaki, H. Okushi, A. Matsuda, K. Tanaka, and J. Isoya, Phys. Rev. Lett. 65, 756 (1990).

${ }^{20}$ N. Orita, T. Matsumura, and H. Katayama-Yoshida, J. Non-Cryst. Solids 198-200, 347 (1996).

${ }^{21}$ S. Yamasaki, S. Kuroda, J. Isoya, and K. Tanaka, J. Non-Cryst. Solids 97-98, 691 (1987).

${ }^{22}$ T. Umeda, S. Yamasaki, J. Isoya, A. Matsuda, and K. Tanaka, Phys. Rev. Lett. 77, 4600 (1996).

${ }^{23}$ S. Yamasaki, Electrotecnical Laboratory Research Report No. 883, 1987 (unpublished).

${ }^{24}$ J. Isoya, S. Yamasaki, A. Matsuda, and K. Tanaka, Philos. Mag. B 69, 263 (1994).

${ }^{25}$ J. Isoya, S. Yamasaki, H. Okushi, A. Matsuda, and K. Tanaka, Phys. Rev. B 47, 7013 (1993).

${ }^{26}$ M. Stutzmann and D. K. Biegelsen, Phys. Rev. B 28, 6256 (1983).

${ }^{27}$ Under illumination, $T_{1}$ of $g=2.0055$ can be decreased due to conversion between neutral and charged DB states, and also due to the cross relaxation between 2.0055 and 2.01 spins, which were discussed in Refs. 26 and 28, respectively.

${ }^{28}$ M. K. Bowman and L. Kevan, in Time Domain Electron Spin Resonance, edited by L. Kevan and R. N. Schwartz (Wiley, New York, 1979), pp. 67-106.
${ }^{29}$ G. Schumm, W. B. Jackson, and R. A. Street, Phys. Rev. B 48, 14198 (1993).

${ }^{30}$ T. Umeda, S. Yamasaki, J. Isoya, A. Matsuda, and K. Tanaka, J. Non-Cryst. Solids 227-230, 353 (1998).

${ }^{31}$ P. C. Taylor, J. F. Baugher, and H. M. Kriz, Chem. Rev. 75, 203 (1975).

${ }^{32}$ H. Dersh, J. Stuke, and J. Beichler, Phys. Status Solidi B 107, 307 (1981).

${ }^{33}$ G. D. Watkins and J. W. Corbett, Phys. Rev. A 134, 1359 (1964).

${ }^{34}$ M. Stutzmann, W. B. Jackson, and C. C. Tsai, Phys. Rev. B 32, 23 (1985).

${ }^{35}$ W. B. Jackson, S. M. Kelso, C. C. Tsai, J. W. Allen, and S.-J. Oh, Phys. Rev. B 31, 5187 (1985).

${ }^{36}$ K. Takeda, K. Shiraishi, M. Fujiki, M. Kondo, and K. Morigaki, Phys. Rev. B 50, 5171 (1994).

${ }^{37}$ N. Ishii and T. Shimizu, Solid State Commun. 102, 647 (1997).

${ }^{38}$ A. J. Stone, Proc. R. Soc. London, Ser. A 271, 424 (1963).

${ }^{39}$ T. Kamei, N. Hata, A. Matsuda, T. Uchiyama, S. Amano, K. Tsukamoto, Y. Yoshioka, and T. Hirao, Appl. Phys. Lett. 68, 2380 (1996).

${ }^{40}$ C. Tsang and R. A. Street, Phys. Rev. B 19, 3027 (1979).

${ }^{41}$ M. Yoshida and K. Morigaki, J. Phys. Soc. Jpn. 58, 3371 (1989).

${ }^{42}$ F. Boulitrop, Phys. Rev. B 28, 6192 (1983).

${ }^{43}$ D. Weaire, N. Higgins, P. Moore, and I. Marshall, Philos. Mag. B 40, 243 (1979).

${ }^{44}$ H. Takenaka, C. Ogihara, and K. Morigaki, J. Phys. Soc. Jpn. 57, 3858 (1988).

${ }^{45}$ J. H. Zhou, S. Yamasaki, J. Isoya, K. Ikuta, M. Kondo, A. Matsuda, and K. Tanaka, in Advances in Microcrystalline and Nanocrystalline Semiconductors-1996, edited by R. W. Collins, P. M. Fauchet, I. Shimizu, J.-C. Vial, T. Shimada, and P. A. Alivisatos, MRS Symposia Proceedings No. 452 (Materials Research Society, Pittsburgh, 1997), p. 821.

${ }^{46}$ N. Ishii, M. Kumeda, and T. Shimizu, Phys. Status Solidi B 116, 91 (1983).

${ }^{47}$ F. Yonezawa and M. H. Cohen, in Fundamental Physics of Amorphous Semiconductors, edited by F. Yonezawa (SpringerVerlag, Singapore, 1981), pp. 119-144. 